\title{
The nature and origin of
}

ultra high-energy

cosmic rays

J.R. Hörandel - Radboud University Nijmegen, the Netherlands

for the Pierre Auger Collaboration, Malargue, Argentina

DOI: 10.1051/epn/2012302

n (for full list of authors, see www.auger.org/admin/Collaborators/author_list_alphabetical.html)

The Earth is exposed to a permanent rain of cosmic particles from ${ }^{\circ}$ outer space [1]. Most of the particles are fully ionised atomic nuclei, moving with relativistic energies. The bulk of them with energies up to $10^{17} \mathrm{eV}$ originate within our Milky Way. They are most likely accelerated in supernova remnants. Some particles have a thousand times higher energies, i.e., around $10^{20} \mathrm{eV}$. This corresponds to the kinetic energy of a tennis ball with a mass of $50 \mathrm{~g}$ and a velocity of $90 \mathrm{~km} / \mathrm{h}$. 
T工 owever, in the tennis ball the energy is distributed between about $10^{24}$ atomic nuclei, while in cosmic rays the energy is concentrated on a single nucleus. This illustrates the immense energies of the highest-energy particles in the Universe. They exceed the energies reached in terrestrial, man made accelerators (like the Large Hadron Collider LHC in Geneva) by a factor of a hundred million.

\section{Possible origin}

The energy density contained in the flux of extragalactic cosmic rays can be inferred from the measured all-particle energy spectrum, see below. To sustain the observed all-particle flux at energies exceeding $10^{17} \mathrm{eV}$, an energy density of $3.7 \times 10^{-7} \mathrm{eV} / \mathrm{cm}^{3}$ is required from cosmic rays originating beyond our galaxy [2]. The power required for a population of sources to generate this energy density over the Hubble time of $10^{10}$ years (the age of the Universe) is $5.5 \times 10^{30} \mathrm{~W} / \mathrm{Mpc}^{3}$ (cf. glossary). Assuming a typical number density for classes of astrophysical sources in the Universe, this number can be converted into an average energy output per source. This leads to about $2 \times 10^{37} \mathrm{~W}$ per active galaxy or approximately $2 \times 10^{45} \mathrm{~W}$ per cosmological gamma ray burster. The coincidence between these numbers and the observed output in electromagnetic energy of these sources explains why they are considered promising candidates to accelerate highest-energy cosmic rays.

The cores of active galaxies are particularly interesting candidates. It is assumed that these galaxies host a black hole in their centre. The black hole attracts matter in an accretion disc. A fraction of this matter is ejected perpendicular to the disc, in the direction of the poles of the system, in a collimated particle stream at relativistic velocities - a jet. Astrophysical calculations show that particles can be accelerated to extremely high energies in such jets. In this scenario, these highest-energy particles propagate through the intergalactic space into our Milky Way and finally the Solar System, where we measure them at Earth.

The Universe is filled with leftover photons from the Big Bang. Each cubic centimetre contains about 400 photons with a radiation temperature of about three Kelvin (the $3 \mathrm{~K}$ background radiation or cosmic microwave background). The cosmic-ray particles lose energy through photo-hadronic interactions with these photons in the "GZK effect" [3]. It occurs at energies exceeding $6 \times 10^{19} \mathrm{eV}$. As a consequence, particles can travel only a limited distance in an otherwise mostly empty/transparent Universe. In other words, the sources of the particles must be located inside a sphere around the Earth. The range of protons with energies around $10^{20} \mathrm{eV}$ amounts to about $100 \mathrm{Mpc}$.

To clarify the origin of the highest-energy particles, their properties have to be measured. Our investigations focus on three main features: energy and arrival direction of the particles plus the particle type (photons, protons, atomic nuclei). The highest-energy cosmic rays are extremely rare.At Earth about

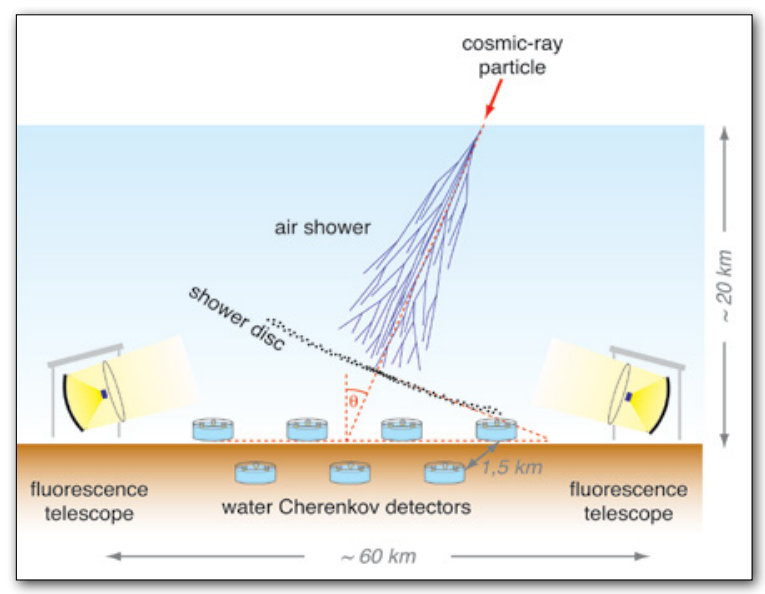

$\triangle$ FIG. 1: Schematic view of the Pierre Auger Observatory

one particle is registered in an area of 100 square kilometres in a hundred years. To measure such particles requires a huge measurement device which is operated for a long time. Such detectors are constructed on the surface of the Earth, rather than in space. The primary cosmic rays themselves are not measured, but secondary particles induced by them in the atmosphere, forming 'extensive air showers'.

\section{The Pierre Auger Observatory}

The Pierre Auger Observatory combines two complementary techniques to measure air showers. On their way through the atmosphere the secondary particles stimulate nitrogen molecules in the air to emit fluorescence light. This light is measured with large telescopes. In addition, secondary particles reaching ground level are registered in an array of particle detectors. The latter are water Cherenkov detectors, measuring the light emitted by relativistic particles passing through a water tank. The set-up is sketched in Fig. 1. The world's largest cosmic-ray detector is located in Argentina, close to the city of Malargue at 1400 metres above sea level. It covers an area of 3000 square kilometres of Pampa. The Observatory has a hexagonal footprint with a diameter of about 60 kilometres.

\section{EXTENSIVE AIR SHOWERS}

In an extensive air shower the kinetic energy of elementary particles is used to produce new particles. According to the famous Einstein formula, $E=m c^{2}$, energy $(E)$ can be converted into mass $(m)-c$ is the speed of light. When a high-energy particle impinges onto the Earth's atmosphere it hits an atomic nucleus of an air molecule. New particles are produced with again extremely high energies. They also interact and a cascade of secondary particles is produced, an extensive air shower. Billions of secondary particles can be produced from the energy of a single cosmic-ray. The secondary particles move in a flat disc, with a thickness of only a few metres, at almost the speed of light through the atmosphere, see Fig. 1. The number of particles in a cascade first increases exponentially as the initial energy is distributed over more and more particles. When the (average) energy per particle in the shower falls below a critical energy, no further particle multiplication is possibleand the number of particles in the cascade decreases due to absorption in the atmosphere. The point at which the shower contains a maximum number of particles is known as the shower maximum. 


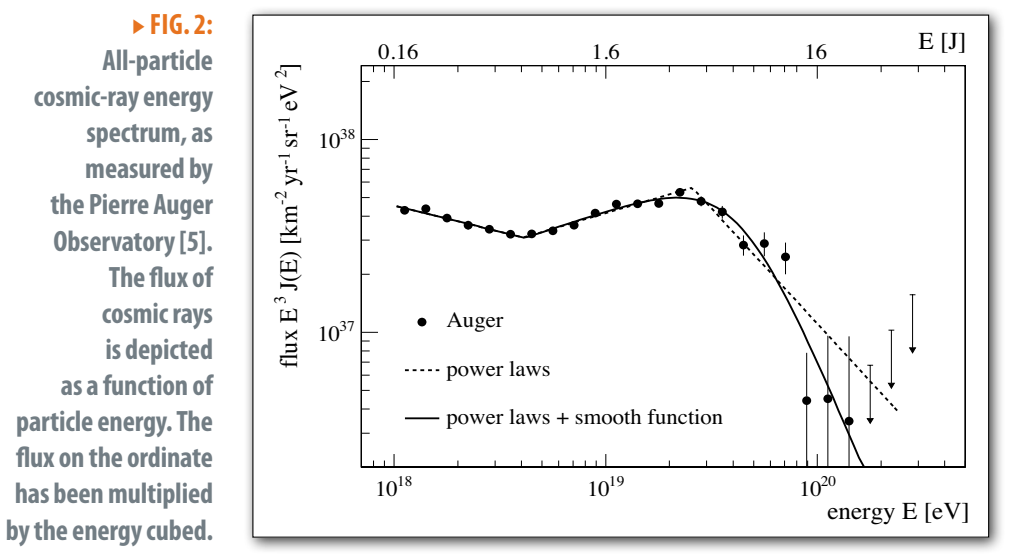

The highly sensitive telescopes can register air showers at distances of several tens of kilometres. This implies that air showers can be observed simultaneously by more than one telescope. The extension of the path of the original particle yields the axis around which the air shower develops. This can be measured to infer the arrival direction of the cosmic-ray particle.

\section{Recent results}

\section{Energy spectrum}

For the measurement of the energy spectrum, i.e., the flux of the incoming cosmic particles as function of their energy, it is crucial to establish an absolute energy scale. The fluorescence light emitted from electrons during their passage through air has been measured in the laboratory at accelerators [4]. This is our "yardstick" to calibrate the energy scale of the observed fluorescence light in an air shower. Since the fluorescence telescopes can be operated only in moonless nights, a second cross-calibration step is necessary. Air showers which are observed simultaneously by the fluorescence telescopes and the surface detectors are used to establish an absolute energy scale for air showers registered only by the surface detectors. Finally, the flux of registered particles can be plotted as function of energy as shown in Figure 2 [5].

\section{One recognises two structures in the spectrum:}

- A dip around $4 \times 10^{18} \mathrm{eV}$. This is believed to be caused by interactions between the cosmic particles and the photons of the $3 \mathrm{~K}$ microwave background. The cosmic rays lose energy due to the production of electron-positron pairs. - A fall-off at energies exceeding several $10^{19} \mathrm{eV}$. There are presently two possible explanations for this feature:

eV, electronvolt, energy unit, $1 \mathrm{eV}=1.6 \times 10^{-19}$ Joule hadron, a hadron is composed of quarks (examples for hadrons are protons and neutrons)

light year, distance unit, 1 light year $=9.4605284 \times 10^{15} \mathrm{~m}$

muon, elementary particle with properties similar to an electron and a mass of 200 electron masses

Mpc, mega parsec, distance unit, $1 \mathrm{Mpc}=3.08 \times 10^{22} \mathrm{~m}$

pion, elementary particle composed of two quarks
1. The cosmic accelerators accelerate particles up to a maximum energy, which is proportional to the magnetic field in the astrophysical source times the charge of the corresponding particle. Thus, one expects that heavy nuclei (with larger atomic number) are accelerated to higher energies and the mass composition of cosmic rays is expected to become heavier with increasing energy.

2. As mentioned before, at energies exceeding several $10^{19} \mathrm{eV}$ cosmic rays interact with the photons of the $3 \mathrm{~K}$ microwave background (GZK effect). Thus, cosmic rays lose a significant fraction of their energy due to photonpion production. Nuclei break up through the GZK effect and it is expected that the composition of cosmic rays is modified during the propagation process.

\section{Mass composition}

The identification of the type of the impinging cosmicray particle is experimentally the biggest challenge. Incoming particles such as atomic nuclei (of different masses), photons, and neutrinos induce cascades in the atmosphere. The longitudinal development of the showers depends on the particle type. This is the key to measuring the particle species with an air shower experiment. Heavy nuclei interact early in the atmosphere, while light particles penetrate much deeper. This implies that for heavy nuclei the whole shower development takes place higher up in the atmosphere as compared to light particles. Thus, a measurement of the height of the shower above ground is a good estimate for the mass of the primary particle. Technically, we measure the distance between the detector and the position at which the shower contains its maximum number of particles. The investigations indicate that cosmic rays are composed of light particles (such as protons and helium nuclei) at energies around $10^{18} \mathrm{eV}$. The data exhibit a trend towards heavier nuclei with increasing energy. At energies around $4 \times 10^{19} \mathrm{eV}$, shower properties consistent with a heavy elemental composition (e.g. silicon or iron nuclei) are observed [6]. At higher energies, at present, no mass measurement is available due to the small flux of particles at such energies. These mass measurements do assume that we can correctly extrapolate hadronic physics from accelerator experiments.

In addition to nuclei, the Auger Observatory is also sensitive to photons and neutrinos [7]. No such particles have been detected yet and thus, upper limits have been established: at energies exceeding $10^{18} \mathrm{eV}$ less than a few percent of the measured particles are photons or neutrinos.

\section{Arrival directions}

In practice, the measurement of the arrival direction is the smallest experimental issue. However, a correct astrophysical interpretation of the data is a big challenge since charged particles are deflected in magnetic fields in the Milky Way and in intergalactic space. 
The Auger Observatory provides two complementary approaches to determine the direction of an incoming cosmic ray. Stereo observations of the showers with multiple fluorescence telescopes provide a three dimensional picture of the shower in the atmosphere and, thus, the orientation of the shower axis, pointing back into the direction of the incoming particle. Secondly, the measurement of the arrival times of the individual particles at the surface detectors allows to measure the shower disc, with the arrival direction being perpendicular to it, see also Fig. 1.

A sky map of the arrival directions of the highest energy particles, with energies exceeding $6 \times 10^{19} \mathrm{eV}$ is shown in Fig. 3 [8].Statistical investigations show that the particles do not arrive isotropically at Earth. The arrival direction of cosmic rays seems to be correlated with the general distribution of matter in the Universe. Several particles seem to originate close to the direction of Centaurus A, a nearby active galactic nucleus, only about 12 million light years from the Earth. The deflection of the particles in magnetic fields in the Universe is proportional to the charge of the particles. At the highest energies the average deflection amounts to about 2.7 degrees times the particle's charge. Hence, a possible correlation of the arrival directions of cosmic rays with astrophysical objects can be most easily explained by low-charge (i.e. light nuclei) cosmic rays.

\section{Conclusions}

The Pierre Auger Observatory provides the most accurate measurements of highest-energy cosmic rays to date. However, the origin of the highest energy particles in the Universe cannot yet be established beyond doubt.

If the observed fall-off in the energy spectrum is due to the GZK effect, the composition observed at Earth depends on the mix of nuclei being emitted from the sources and modifications to it during the propagation through the Universe. In case of a light composition (small charge), the particles would be only marginally deflected in magnetic fields and the particle trajectories would point back to their sources.

If the fall-off in the energy spectrum is caused by the maximum energy attained in astrophysical accelerators, this would yield a heavy composition at Earth (large charge) at the highest energies, as seen by a trend in the observed mass composition. In this case an interpretation of the arrival directions of cosmic rays is more difficult. A possible key to resolve this issues are detailed studies of hadronic interactions in the atmosphere such as, e.g., the measurements of the proton-proton interaction cross sections at energies exceeding the energies of the LHC [9] and direct measurements of the interaction parameters at the LHC. Such data will further improve our understanding of the development of air showers in the atmosphere. With this increased knowledge we hope to clarify the origin of the highest energy particles in the Universe in the near future.

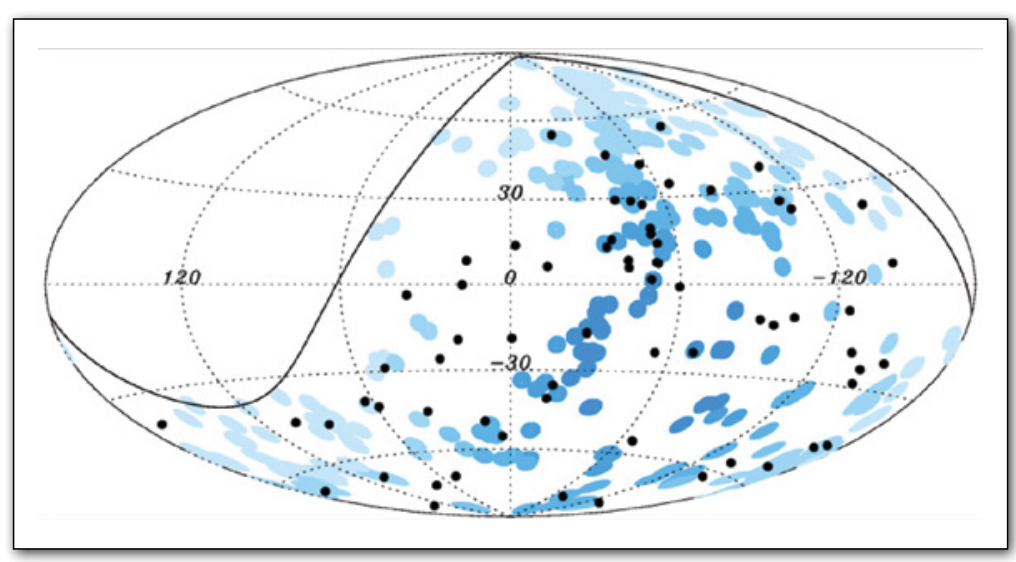

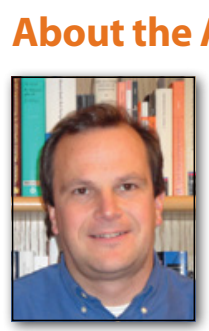

Jörg Hörandel studied Physics at the University of Karlsruhe, Germany, where he also obtained his $\mathrm{PhD}$ in 1997 and later his Habilitation/Venia Legendi in 2004. Since 2007 he is assistant professor of astroparticle physics and astronomy at Radboud University Nijmegen, the Netherlands.

\section{Acknowledgement}

The Pierre Auger Observatory has been built and is operated by scientists from many countries around the world, see www.auger.org and www.auger.org/admin/Collaborators/acknowledgments.pdf.

The author would like to thank Roger Clay for his valuable comments on the manuscript.

\section{References}

[1] M. Nagano, A.A. Watson, Rev. Mod. Phys. 72, 689 (2000); J. Blümer, R. Engel, J.R. Hörandel, Progress in Particle and Nuclear Physics 63, 293 (2009).

[2] J.R. Hörandel, Reviews in Modern Astronomy 20, 203 (2008) and references therein

[3] K. Greisen, Phys. Rev. Lett. 16, 748 (1966); G.T. Zatsepin, V.A. Kuz'min, JETP Lett. 4, 78 (1966).

[4] F. Arqueros, J.R. Hörandel, B. Keilhauer, Nuclear Instruments and Methods A 597, 1 (2008).

[5] J. Abraham et al. (Pierre Auger Collaboration), Physics Letters $B$ 685, 239 (2010); J. Abraham et al. (Pierre Auger Collaboration), Physical Review Letters 101, 061101 (2008).

[6] J. Abraham et al. (Pierre Auger Collaboration), Physical Review Letters 104, 091101 (2010).

[7] J. Abraham et al. (Pierre Auger Collaboration), Astroparticle Physics 31, 399 (2009) ; J. Abraham et al. (Pierre Auger Collaboration), Physical Review D 79, 102001 (2009).

[8] J. Abraham et al. (Pierre Auger Collaboration), Science 318, 938 (2007); J. Abraham et al. (Pierre Auger Collaboration), Astroparticle Physics 29, 188 (2008); P. Abreu et al. (Pierre Auger Collaboration), Astroparticle Physics 34, 314 (2010).

[9] R. Ulrich et al. (Pierre Auger Collaboration), Proceedings of the $32^{\text {nd }}$ International Cosmic Ray Conference, Beijing, 946 (2011). $\triangle F I G$. 3: Arrival direction of the highest energy cosmic rays $(\mathrm{E}>$ $6 \times 10^{19} \mathrm{eV}$ ) on the sky, as measured by the Pierre Auger Observatory [8]. The black dots indicate the measured cosmic rays. The blue circles represent the positions of active galactic nuclei (AGN), the intensity is proportional to the fraction of time during which the particular source can be seen by the Auger Observatory. The map is in Galactic coordinates. 